\title{
Analysis of Robust Stability for a Class of Stochastic Systems via Output Feedback: The LMI Approach
}

\author{
Xin-rong Cong and Long-suo Li \\ Department of Mathematics, Harbin Institute of Technology, Harbin 150001, China \\ Correspondence should be addressed to Long-suo Li; lls@hit.edu.cn
}

Received 4 August 2013; Accepted 1 November 2013

Academic Editor: Shusen Ding

Copyright ( 12013 X.-r. Cong and L.-s. Li. This is an open access article distributed under the Creative Commons Attribution License, which permits unrestricted use, distribution, and reproduction in any medium, provided the original work is properly cited.

\begin{abstract}
This paper investigates the robust stability for a class of stochastic systems with both state and control inputs. The problem of the robust stability is solved via static output feedback, and we convert the problem to a constrained convex optimization problem involving linear matrix inequality (LMI). We show how the proposed linear matrix inequality framework can be used to select a quadratic Lyapunov function. The control laws can be produced by assuming the stability of the systems. We verify that all controllers can robustly stabilize the corresponding system. Further, the numerical simulation results verify the theoretical analysis results.
\end{abstract}

\section{Introduction}

The stochastic differential equations which play an important role in many branches of science and industry are often used as the tool of describing complex phenomena $[1,2]$. The stochastic items of the stochastic differential equations lead to the solutions of the equations also with randomness, so the stability analysis is very crucial. Roughly speaking, the stability means insensitivity of the state of the system to small changes in the parameters or the initial state of the system [3]. In this paper, the robust stability for a class of stochastic systems is investigated via static output feedback by using the unified linear matrix inequalities approach.

The problem of robust stability [4-6] refers to the control system under the existence of internal uncertainty and external disturbance; static or dynamic feedback controller is designed to stabilize the closed-loop system. When the uncertainty factors exist in the system, the robust stability is the indicator of the system which can still maintain normal work performance.

Many important problems in stochastic differential equation theory can be converted to convex optimization problems with linear matrix inequalities, so that they will become numerically tractable. The quadratic stability theory of discrete-time systems has been paid much attention, which can be seen in [7-10]. The robust quadratic stabilization of nonlinear systems within the framework of linear matrix inequalities is investigated by Šiljak and Stipanović [11]. Further, the robust stabilization for a class of discrete-time nonlinear systems is formulated into a convex optimization via linear matrix inequalities developed by Stipanović and Śiljak [12]. Then, Ho and Lu [13] who extended the work of Stipanović and Šiljak investigated the robust stabilization for a class of discrete-time nonlinear systems via output feedback. Based on these works, we consider a class of stochastic systems. The major objective of this paper is to show a method to design static output feedback controller for a class of stochastic systems by means of convex optimization problems via linear matrix inequalities; then, the static output feedback control laws can robustly stabilize the stochastic systems.

In this paper, we suppose that $(\Omega, F, P)$ is a complete probability space with a filtration $\left\{F_{t}\right\}_{t \geq 0}$ satisfying the usual conditions. $R^{n}$ and $R^{m \times n}$ denote the $n$-dimension Euclidean space and the set of all $m \times n$ real matrices, respectively. $A^{T}$ represents the transpose of the matrix $A$, and $A^{+}$represents the generalized inverse matrix of $A$. $I$ denotes the identity matrix. $L^{p}\left(R_{+} ; R^{d}\right)$ represents the family of $R^{d}$ valued random variables $X$ with $E|X|^{p}<\infty$. $C^{2,1}\left(R^{n} \times R_{+} ; R_{+}\right)$represents 
the family of all real-valued functions $V(x(t), t)$ defined on $R^{n} \times R_{+}$which are continuously twice differentiable in $x \in R^{n}$ and once differentiable in $t \in R_{+}$.

\section{Preliminaries}

Let us introduce some definitions and lemmas which are needed to prove our main results of this paper.

Definition 1 (see [14]). A linear matrix inequality can be represented as the following form:

$$
F(x):=F_{0}+\sum_{i=1}^{n} x_{i} F_{i}>0
$$

where $x=\left\{x_{1}, x_{2}, \ldots, x_{n}\right\}$ is the decision vector and $\left\{F_{i}, \quad(i=\right.$ $0,1,2, \ldots, n)\}$ are given symmetric matrix.

Lemma 2 (Schur complement [15]). For the given symmetric matrix $S=\left[\begin{array}{ll}S_{11} & S_{12} \\ S_{12}^{T} & S_{22}\end{array}\right]$, then, one has the following equivalent conditions:

(a) $S<0$;

(b) $S_{11}<0, S_{22}-S_{12}^{T} S_{11}^{-1} S_{12}<0$;

(c) $S_{22}<0, S_{11}-S_{12} S_{22}^{-1} S_{12}^{T}<0$.

Lemma 3 (see [13]). Let $\Omega_{1}(x)$ and $\Omega_{2}(x)$ be two arbitrary quadratic forms over $R^{n}$; then $\Omega_{1}(x)<0$ for all $x \in R^{n}-\{0\}$ satisfying $\Omega_{2}(x) \leq 0$ if and only if there exists $\tau \geq 0$ such that

$$
\Omega_{1}(x)-\tau \Omega_{2}(x)<0, \quad \forall x \in R^{n}-\{0\} .
$$

Lemma 4 (the multidimensional Itô formula [16]). Let $x(t)$ be a d-dimensional Ito process on $t \geq 0$ with the stochastic differential

$$
d x(t)=f(t) d t+g(t) d W(t)
$$

where $f \in L^{1}\left(R_{+} ; R^{d}\right), g \in L^{2}\left(R_{+} ; R^{d \times m}\right)$, and $W(t)$ is an $m$ dimensional Brownian motion. Let $V \in C^{2,1}\left(R^{d} \times R_{+} ; R\right)$. Then $V(x(t), t)$ is again an Itô process with stochastic differential given by

$$
\begin{aligned}
d V(x(t), t)= & {\left[V_{t}(x(t), t)+V_{x}(x(t), t) f(t)\right.} \\
& \left.+\frac{1}{2} \operatorname{trace}\left(g^{T}(t) V_{x x}(x(t), t) g(t)\right)\right] d t \\
& +V_{x}(x(t), t) g(t) d B(t) .
\end{aligned}
$$
[16]:

Consider the following stochastic differential equation

$$
d x(t)=f(t) d t+g(t) d W(t) .
$$

Define the differential operator $L$ associated with (5) by

$$
\begin{aligned}
L= & \frac{\partial}{\partial t}+\sum_{i=1}^{n} f_{i}(x, t) \frac{\partial}{\partial x_{i}} \\
& +\frac{1}{2} \sum_{i, j=1}^{n}\left[g(x, t) g^{T}(x, t)\right]_{i j} \frac{\partial^{2}}{\partial x_{i} x_{j}} .
\end{aligned}
$$

If $L$ acts on a function $V \in C^{2,1}\left(R^{n} \times R_{+} ; R_{+}\right)$, then

$$
\begin{aligned}
L V(x, t)= & V_{t}(x, t)+V_{x}(x, t) f(x, t) \\
& +\frac{1}{2} \operatorname{trace}\left[g^{T}(x, t) V_{x x}(x, t) g(x, t)\right] .
\end{aligned}
$$

We set

$$
\begin{gathered}
V_{t}=\frac{\partial V}{\partial t}, \quad V_{x}=\left(\frac{\partial V}{\partial x_{1}}, \frac{\partial V}{\partial x_{2}}, \ldots, \frac{\partial V}{\partial x_{n}}\right), \\
V_{x x}=\left(\frac{\partial^{2} V}{\partial x_{i} \partial x_{j}}\right)_{n \times n}=\left(\begin{array}{ccc}
\frac{\partial^{2} V}{\partial x_{1} \partial x_{1}} & \cdots & \frac{\partial^{2} V}{\partial x_{1} \partial x_{n}} \\
\vdots & & \vdots \\
\frac{\partial^{2} V}{\partial x_{n} \partial x_{1}} & \cdots & \frac{\partial^{2} V}{\partial x_{n} \partial x_{n}}
\end{array}\right) .
\end{gathered}
$$

Then, we have the following lemma.

Lemma 5 (see [16]). If there exists a positive-definite decrescent function $V \in C^{2,1}\left(R^{n} \times R_{+} ; R_{+}\right)$such that

$$
L V(x, t)<0,
$$

then the trivial solution of (5) is stochastically asymptotically stable.

\section{Main Results}

Consider the following stochastic system:

$$
d x(t)=[A x(t)+f(t, x(t))] d t+D x(t) d w(t),
$$

where $x(t) \in R^{n}$ is the state of the system, $A \in R^{n \times n}$ and $D \in R^{n \times n}$ are constant matrix with appropriate dimensions, and $w(t)$ is one-dimensional Brownian motion which satisfies

$$
E[d w(t)]=0, \quad E\left[d w^{2}(t)\right]=d t .
$$

$f(t, x(t))$ is a known continuous vector-valued Borel measurable function and satisfies the following quadratic inequality:

$$
f^{T}(t, x(t)) f(t, x(t)) \leq \alpha^{2} x^{T} F^{T} F x,
$$

which can be rewritten as

$$
\left[\begin{array}{l}
x \\
f
\end{array}\right]^{T}\left[\begin{array}{cc}
-\alpha^{2} F^{T} F & 0 \\
0 & I
\end{array}\right]\left[\begin{array}{l}
x \\
f
\end{array}\right] \leq 0
$$

where $\alpha$ is nonnegative constant and $F$ is a constant matrix with appropriate dimension. 
Remark 6. For any given $F$, we note that inequality (12) defines a class of functions

$$
\begin{aligned}
F_{\alpha}=\{f & : R^{n+1} \longrightarrow R^{n} \mid f^{T} f \\
& \left.\leq \alpha^{2} x^{T} F^{T} F x,(t, x) \in R_{+} \times R^{n}\right\} .
\end{aligned}
$$

$F_{\alpha}$ includes function $F(t, 0)=0$, and $x=0$ corresponding to the initial value $x\left(t_{0}\right)=0$ is a trivial solution of system (10). For the convenience of the discussion, let $\gamma=\alpha^{-2}$.

Definition 7. System (10) is robustly stable with degree $\alpha$ if the trivial solution $x=0$ is stochastically asymptotically stable for all $f(t, x(t))$ satisfying constraint (12).

Theorem 8. System (10) is robustly stable with degree $\alpha$ if there exists positive-definite matrix $Y$ such that the following convex optimization problem is solvable:

minimize $\gamma$

subject to $Y>0$

$$
\left[\begin{array}{cccc}
Y A^{T}+A Y & I & Y D^{T} & Y F^{T} \\
I & -I & 0 & 0 \\
D Y & 0 & -Y & 0 \\
F Y & 0 & 0 & -\gamma I
\end{array}\right]<0
$$

where $\gamma=\alpha^{-2}$.

Proof. In order to establish robust stability in the sense of Definition 7, the following quadratic Lyapunov function is used:

$$
V(x, t)=x^{T} Q x,
$$

where $Q$ is a symmetric positive-definite matrix $(Q>0)$. According to (7), when we compute $\operatorname{LV}(x, t)$ with respect to system (10), we have

$$
\begin{aligned}
L V(x, t)= & 2 x^{T}(t) \cdot Q \cdot[A x(t)+f(t, x(t))] \\
& +x^{T}(t) D^{T} \cdot Q \cdot D x(t) \\
= & 2 x^{T}(t) \cdot Q \cdot A x(t)+2 x^{T}(t) \cdot Q \cdot f(t, x(t)) \\
& +x^{T}(t) D^{T} \cdot Q \cdot D x(t) \\
= & x^{T}(t) \cdot Q A \cdot x(t)+x^{T}(t) \cdot A^{T} Q \cdot x(t) \\
& +x^{T}(t) \cdot Q \cdot f(t, x(t)) \\
& +f^{T}(t, x(t)) \cdot Q \cdot x(t)+x^{T}(t) \cdot D^{T} Q D \cdot x(t) .
\end{aligned}
$$

From Lemma 5, for the stochastically asymptotically stability, we have

$$
L V(x, t)<0
$$

We rewrite (17) as

$$
\left[\begin{array}{l}
x \\
f
\end{array}\right]^{T}\left[\begin{array}{cc}
Q A+A^{T} Q+D^{T} Q D & Q \\
Q & 0
\end{array}\right]\left[\begin{array}{l}
x \\
f
\end{array}\right]<0 .
$$

From Lemma 3 we further rewrite (19) using (13) as the inequalities

$$
\left[\begin{array}{l}
x \\
f
\end{array}\right]^{T}\left[\begin{array}{cc}
Q A+A^{T} Q+D^{T} Q D+\tau \alpha^{2} F^{T} F & Q \\
Q & -\tau I
\end{array}\right]\left[\begin{array}{l}
x \\
f
\end{array}\right]<0
$$

where $P>0$ and $\tau \geq 0$. Namely, we have the following inequalities:

$$
\left[\begin{array}{cc}
\mathrm{Q} A+A^{T} \mathrm{Q}+D^{T} \mathrm{Q} D+\tau \alpha^{2} F^{T} F & \mathrm{Q} \\
\mathrm{Q} & -\tau I
\end{array}\right]<0 .
$$

Further, from Lemma 2, we rewrite (21) as

$$
\left[\begin{array}{ccc}
Q A+A^{T} Q+\tau \alpha^{2} F^{T} F & Q & D^{T} \\
Q & -\tau I & 0 \\
D & 0 & -Q^{-1}
\end{array}\right]<0
$$

Because $\tau \geq 0$, the inequalities (22) represent nonstrict linear matrix inequalities. From the known result of [14], if there is a solution for $\tau=0$, there is a solution for some $\tau>0$ and sufficiently small $\alpha$; then, we can replace $\tau \geq 0$ by $\tau>0$. Multiplying the first row and the first column of matrix (22) by $Q^{-1}$, we have

$$
\left[\begin{array}{ccc}
Q^{-1} A^{T}+A Q^{-1}+\tau \alpha^{2} Q^{-1} F^{T} F Q^{-1} & I & Q^{-1} D^{T} \\
I & -\tau I & 0 \\
D Q^{-1} & 0 & -Q^{-1}
\end{array}\right]<0 .
$$

Multiplying the first row and the third row of matrix (23) by $\tau$, we have

$$
\left[\begin{array}{ccc}
\tau Q^{-1} A^{T}+\tau A Q^{-1}+\tau \alpha^{2} Q^{-1} F^{T} F \tau Q^{-1} & \tau I & \tau Q^{-1} D^{T} \\
I & -\tau I & 0 \\
\tau D Q^{-1} & 0 & -\tau Q^{-1}
\end{array}\right]<0 .
$$

Let $Y=\tau Q^{-1}$; therefore, $(24)$ is further equivalent to

$$
\left[\begin{array}{ccc}
Y A^{T}+A Y+\alpha^{2} Y F^{T} F Y & \tau I & Y D^{T} \\
I & -\tau I & 0 \\
D Y & 0 & -Y
\end{array}\right]<0 .
$$

Dividing the second column of the matrix (25) by $\tau$, we have

$$
\left[\begin{array}{ccc}
Y A^{T}+A Y+\alpha^{2} Y F^{T} F Y & I & Y D^{T} \\
I & -I & 0 \\
D Y & 0 & -Y
\end{array}\right]<0
$$

From Lemma 2, we rewrite (26) as

$$
Y>0
$$

$$
\left[\begin{array}{cccc}
Y A^{T}+A Y & I & Y D^{T} & Y F^{T} \\
I & -I & 0 & 0 \\
D Y & 0 & -Y & 0 \\
F Y & 0 & 0 & -\gamma I
\end{array}\right]<0
$$


where $Y=\tau Q^{-1}$. We assume that the matrix $F$ is selected and maximize parameter $\alpha$ by solving the following LMI problem in $Y$ and $\gamma$ :

minimize $\gamma$

subject to $Y>0$

$$
\left[\begin{array}{cccc}
Y A^{T}+A Y & I & Y D^{T} & Y F^{T} \\
I & -I & 0 & 0 \\
D Y & 0 & -Y & 0 \\
F Y & 0 & 0 & -\gamma I
\end{array}\right]<0
$$

The proof is now complete.

Now, we introduce static output feedback to stabilize the system. Consider the following stochastic system:

$$
\begin{gathered}
d x(t)=[A x(t)+B u(t)+f(t, x(t))] d t \\
+[D x(t)+C u(t)] d w(t) \\
y(t)=E x(t),
\end{gathered}
$$

where $x(t) \in R^{n}, u(t) \in R^{m}$, and $y(t) \in R^{p}$ are the system state and control input and output, respectively; $A \in$ $R^{n \times n}, D \in R^{n \times n}, B \in R^{n \times m}, C \in R^{n \times m}$, and $E \in R^{p \times n}$ are constant matrices; without loss of generality, suppose that rank $(E)=p ; f(t, x(t))$ satisfies the constraint condition (12).

Consider the following form of linear static output feedback controller:

$$
u(t)=K y(t),
$$

where $K \in R^{m \times p}$ is a constant matrix to be determined.

Definition 9. If there exists a controller in the form of (30) such that the system (29) and (30) is robustly stable with degree $\alpha$ in the sense of Definition 7, then, we say that system (29) is robustly stabilized with degree $\alpha$ by the control law (30).

Theorem 10. System (29) is robustly stabilizable with degree $\alpha$ by means of static output feedback (30) if the following optimization problem is solvable:

minimize $\gamma$

subject to $Y>0$

$$
\left[\begin{array}{cccc}
Y A^{T}+A Y+L^{T} B^{T}+B L & I & Y D^{T}+L^{T} C^{T} & Y F^{T} \\
I & -I & 0 & 0 \\
D Y+C L & 0 & -Y & 0 \\
F Y & 0 & 0 & -\gamma I
\end{array}\right]<0 .
$$

Proof. Applying the static output feedback (30) to the system (29), we have

$$
\begin{aligned}
d x(x, t)= & {[A x(t)+B K E x(t)+f(t, x(t))] d t } \\
& +[D x(t)+C K E x(t)] d w(t) \\
= & {[(A+B K E) x(t)+f(t, x(t))] d t } \\
& +(D+C K E) x(t) d w(t) \\
\doteq & {[\widehat{A} x(t)+f(t, x(t))] d t+\widehat{D} x(t) d w(t) . }
\end{aligned}
$$

Namely,

$$
d x(t)=[\widehat{A} x(t)+f(t, x(t))] d t+\widehat{D} x(t) d w(t),
$$

where $\widehat{A}=A+B K E$ and $\widehat{D}=D+C K E$. Using quadratic function $V(x)$ and computing $L V(x, t)$ with respect to system (33) which is similar to Theorem 8 , we have the following inequalities:

$$
\left[\begin{array}{cccc}
Y A^{T}+A Y+Y E^{T} K^{T} B^{T}+B K E Y & I & Y D^{T}+Y E^{T} K^{T} C^{T} & Y F^{T} \\
I & -I & 0 & 0 \\
D Y+C K E Y & 0 & -Y & 0 \\
F Y & 0 & 0 & -\gamma I
\end{array}\right]<0,
$$

which is not an LMI in $Y, K$, and $E$ but can be made so by introducing the change of variable

$$
K E Y=L
$$

or

$$
K=L(Y)^{-1} E^{+} .
$$

Since the transformed problem is now of LMI variety, we have minimize $\gamma$ subject to $Y>0$

$$
\left[\begin{array}{cccc}
Y A^{T}+A Y+L^{T} B^{T}+B L & I & Y D^{T}+L^{T} C^{T} & Y F^{T} \\
I & -I & 0 & 0 \\
D Y+C L & 0 & -Y & 0 \\
F Y & 0 & 0 & -\gamma I
\end{array}\right]<0 .
$$

The proof is now complete.
To make the outcomes of problem (31) practical, we have to impose some restrictions on $Y$ and $L . Y$ is restricted to the structure

$$
Y=\left[\begin{array}{ll}
\widetilde{Q}_{1} & o \\
o^{T} & \widetilde{q}_{2}
\end{array}\right]
$$

where $\widetilde{Q}_{1}$ is a $(n-1) \times(n-1)$ matrix, $o \in R^{n-1}$ is a zero vector, and $\widetilde{q}_{2} \in R$.

$L$ is restricted as

$$
L=\left[\begin{array}{l}
O \\
l
\end{array}\right]
$$


where $O$ is an $(n-1) \times n$ zero matrix and $l=\left(l_{1}, l_{2}, \ldots, l_{n}\right)$ is a row vector. Now, we propose the minimization problem in order to maximize $\alpha$,

minimize $\gamma$

subject to $Y>0$

$$
\left[\begin{array}{cccc}
Y A^{T}+A Y+L^{T} B^{T}+B L & I & Y D^{T}+L^{T} C^{T} & Y F^{T} \\
I & -I & 0 & 0 \\
D Y+C L & 0 & -Y & 0 \\
F Y & 0 & 0 & -\gamma I
\end{array}\right]<0,
$$

where $Y$ and $L$ have the form described in (38) and (39). If the optimization problem is feasible, we can compute $K E$ by

$$
K E=l \cdot \widetilde{q}_{2}^{-1}=\left(\frac{l_{1}}{\widetilde{q}_{2}}, \frac{l_{2}}{\widetilde{q}_{2}}, \ldots, \frac{l_{n}}{\widetilde{q}_{2}}\right) .
$$

Then, we have the following corollary.

Corollary 11. System (29) is robustly stabilizable with degree $\alpha$ by means of static output feedback (30) if the problem (41) is feasible.

We note that it is possible to incorporate constraints on the size of the vector $K$ in optimization problem (40). we can restrict the size of $K$ by constraining $\widetilde{q}_{2}^{-1}$ and $l$ (see [12]). We set

$$
l l^{T}<\kappa_{l}, \quad \kappa_{l}>0
$$

which is equivalent to the LMI

$$
\left[\begin{array}{cc}
-\kappa_{l} & l \\
l^{T} & -I
\end{array}\right]<0
$$

Then, we suppose that

$$
\tilde{q}_{2}^{-1}<\kappa_{q}, \quad \kappa_{q}>0
$$

Inequality (44) can be rewritten as the linear matrix inequalities

$$
\left[\begin{array}{cc}
\kappa_{q} & 1 \\
1 & \tilde{q}_{2}
\end{array}\right]>0
$$

We can get the optimized bound from constraints (42) and (44), which is shown as follows:

$$
(K E)(K E)^{T}=l l^{T} \widetilde{q}_{2}^{-2}<\kappa_{l} \kappa_{q}^{2},
$$

which has the LMI representation (43) and (45).

With these modifications the optimization problem (40) becomes

$$
\begin{aligned}
& \operatorname{minimize} \gamma+\kappa_{l}+\kappa_{q} \\
& \text { subject to } Y>0
\end{aligned}
$$

$$
\begin{aligned}
& {\left[\begin{array}{cccc}
Y A^{T}+A Y+L^{T} B^{T}+B L & I & Y D^{T}+L^{T} C^{T} & Y F^{T} \\
I & -I & 0 & 0 \\
D Y+C L & 0 & -Y & 0 \\
F Y & 0 & 0 & -\gamma I
\end{array}\right]<0} \\
& {\left[\begin{array}{cc}
-\kappa_{l} & l \\
l^{T} & -l
\end{array}\right]<0} \\
& {\left[\begin{array}{cc}
\kappa_{q} & 1 \\
1 & \tilde{q}_{2}
\end{array}\right]>0 .}
\end{aligned}
$$

Then, we have the following corollary.

Corollary 12. System (29) is robustly stabilizable with degree $\alpha$ by means of static output feedback (30) if the problem (47) is feasible.

Further, by expanding the system (29), consider the following stochastic system:

$$
\begin{gathered}
d x(t)=\left[A_{0} x(t)+B_{0} u(t)+f(t, x(t), u(t))\right] d t \\
+\left[D_{0} x(t)+C_{0} u(t)\right] d w(t) \\
y(t)=E_{0} x(t),
\end{gathered}
$$

where $x(t) \in R^{n}, u(t) \in R^{m}$, and $y(t) \in R^{p}$ are the system state and control input and output, respectively; $A_{0} \in$ $R^{n \times n}, D_{0} \in R^{n \times n}, B_{0} \in R^{n \times m}, C_{0} \in R^{n \times m}$, and $E_{0} \in R^{p \times n}$ are constant matrices; without loss of generality, we suppose that rank $\left(E_{0}\right)=p ; f(t, x(t), u(t))$ is known vector-valued function and satisfies the following constraint condition:

$$
f^{T}(t, x, u) f(t, x, u) \leq \alpha^{2}\left(x^{T} H^{T} H x+u^{T} G^{T} G u\right),
$$

where $H$ and $G$ are constant matrices with appropriate dimensions and $\alpha$ is nonnegative constant. 
Consider the following form of linear static output feedback controller:

$$
u(t)=K_{0} y(t)
$$

where $K_{0} \in R^{m \times p}$ is a constant matrix to be determined.

Definition 13. If there exists a controller in the form of (50) such that the system (48) and (50) is robustly stable with degree $\alpha$ in the sense of Definition 7, then, we say that system (48) is robustly stabilized with degree $\alpha$ by the control law (50).

Theorem 14. System (48) is robustly stabilizable with degree $\alpha$ by means of static output feedback (50) if the following optimization problem on $Y \in R^{n \times n}, X \in R^{m \times p}$, and $Z \in R^{p \times p}$ is solvable:

minimize $\gamma$

subject to $Y>0$

$$
\begin{aligned}
& {\left[\begin{array}{cccc}
Y A_{0}^{T}+A_{0} Y+B_{0} X E_{0}+E_{0}^{T} X^{T} B_{0}^{T} & I & Y D^{T}+E_{0}^{T} X^{T} C_{0}^{T} & F_{0}^{T} \\
I & -I & 0 & 0 \\
D_{0} Y+C_{0} X E_{0} & 0 & -Y & 0 \\
F_{0} & 0 & 0 & -\gamma I
\end{array}\right]<0} \\
& E_{0} Y=Z E_{0},
\end{aligned}
$$

where $F_{0}=\left(\begin{array}{c}H Y \\ G X E_{0}\end{array}\right)$.

Proof. Applying the static output feedback (50) to the system (48), we have

$$
\begin{aligned}
d x(x, t)= & {\left[A_{0} x(t)+B_{0} K_{0} E_{0} x(t)+f\left(t, x(t), K_{0} E_{0} x(t)\right)\right] d t } \\
& +\left[D_{0} x(t)+C_{0} K_{0} E_{0} x(t)\right] d w(t) \\
= & {\left[\left(A_{0}+B_{0} K_{0} E_{0}\right) x(t)+f\left(t, x(t), K_{0} E_{0} x(t)\right)\right] d t } \\
& +\left[D_{0}+C_{0} K_{0} E_{0}\right] x(t) d w(t) \\
\doteq & {\left[\widehat{A} x(t)+f\left(t, x(t), K_{0} E_{0} x(t)\right)\right] d t } \\
& +\widehat{D} x(t) d w(t),
\end{aligned}
$$

where $f\left(t, x(t), K_{0} E_{0} x(t)\right)$ can be transformed to

$$
\begin{aligned}
f^{T} & \left(t, x(t), K_{0} E_{0} x(t)\right) f\left(t, x(t), K_{0} E_{0} x(t)\right) \\
& \leq \alpha^{2}\left[x^{T}(t) H^{T} H x(t)+\left(K_{0} E_{0} x(t)\right)^{T} G^{T} G\left(K_{0} E_{0} x(t)\right)\right] \\
& =\alpha^{2}\left[x^{T}(t) H^{T} H x(t)+x^{T}(t) E_{0}^{T} K_{0}^{T} G^{T} G K_{0} E_{0} x(t)\right] \\
& =\alpha^{2} x^{T}(t)\left(\begin{array}{c}
H \\
G K_{0} E_{0}
\end{array}\right)^{T}\left(\begin{array}{c}
H \\
G K_{0} E_{0}
\end{array}\right) x(t) .
\end{aligned}
$$

Namely,

$$
d x(t)=\left[\widehat{A} x(t)+f\left(t, x(t), K_{0} E_{0} x(t)\right)\right] d t+\widehat{D} x(t) d w(t)
$$

where $\widehat{A}=A_{0}+B_{0} K_{0} E_{0}$ and $\widehat{D}=D_{0}+C_{0} K_{0} E_{0}$. That is, system (54) is equivalent to (10) with constraint (12) if

$$
\begin{gathered}
A=\widehat{A}=A_{0}+B_{0} K_{0} E_{0}, \\
D=\widehat{D}=D_{0}+C_{0} K_{0} E_{0}, \\
F=\left(\begin{array}{c}
H \\
G K_{0} E_{0}
\end{array}\right) .
\end{gathered}
$$

Noting that the full row rank of $E$ and $Y$ is a positive-definite matrix from LMI (51), then matrix $E_{0} Y=Z E_{0}$ implies that

$$
\begin{gathered}
p \geq \operatorname{rank}(Z) \geq \operatorname{rank}\left(Z E_{0}\right)=\operatorname{rank}\left(E_{0} Y\right) \\
\geq \operatorname{rank}\left[\left(E_{0} Y\right) \cdot Y^{-1}\right]=\operatorname{rank}\left(E_{0}\right)=p
\end{gathered}
$$

then, $Z$ is nonsingular. If the gain of control law in the form of (50) can be chosen as

$$
K_{0}=X Z^{-1}
$$

then, in LMI (26), we have

$$
\begin{aligned}
A Y & =\left(A_{0}+B_{0} K_{0} E_{0}\right) Y=A_{0} Y+B_{0} K_{0} E_{0} Y \\
& =A_{0} Y+B_{0} K_{0} Z E_{0}=A_{0} Y+B_{0} X E_{0} \\
D Y & =\left(D_{0}+C_{0} K_{0} E_{0}\right) Y=D_{0} Y+C_{0} K_{0} E_{0} Y \\
& =D_{0} Y+C_{0} K_{0} Z E_{0}=D_{0} Y+C_{0} X E_{0}
\end{aligned}
$$

$F Y=\left(\begin{array}{c}H \\ G K_{0} E_{0}\end{array}\right) Y=\left(\begin{array}{c}H Y \\ G K_{0} E_{0} Y\end{array}\right)=\left(\begin{array}{c}H Y \\ G K_{0} Z E_{0}\end{array}\right)=\left(\begin{array}{c}H Y \\ G X E_{0}\end{array}\right)$, 
then, for systems (54), the following LMI is equivalent to the LMI in (15):

$$
\left[\begin{array}{cccc}
Y A_{0}^{T}+A_{0} Y+B_{0} X E_{0}+E_{0}^{T} X^{T} B_{0}^{T} & I & Y D^{T}+E_{0}^{T} X^{T} C_{0}^{T} & \left(Y H^{T}, E_{0}^{T} X^{T} G^{T}\right) \\
I & -I & 0 & 0 \\
D_{0} Y+C_{0} X E_{0} & 0 & -Y & 0 \\
\left(\begin{array}{c}
H Y \\
G X E_{0}
\end{array}\right) & 0 & 0 & -\gamma I
\end{array}\right]<0 .
$$

From Theorem 8 , the system (54) are robustly stable with degree $\alpha$.

The proof is now complete.

\section{Numerical Simulation}

To further verify the flexibility and correctness of the theory in this paper, we present a simple numerical example.

Example 15. We consider the stochastic systems (29) with

$$
\begin{gathered}
A=\left[\begin{array}{cc}
-5 & 1 \\
-2 & -3
\end{array}\right], \quad B=\left[\begin{array}{l}
0 \\
1
\end{array}\right], \quad D=\left[\begin{array}{cc}
1 & 0.1 \\
-0.2 & -0.5
\end{array}\right], \\
C=\left[\begin{array}{l}
1 \\
2
\end{array}\right], \quad F=\left[\begin{array}{ll}
1 & -2
\end{array}\right], \quad E=\left[\begin{array}{ll}
0 & 1
\end{array}\right] .
\end{gathered}
$$

In this example, Theorem 10 is applied to construct a static output feedback law.

By using the Matlab control toolbox to solve the convex optimization problem (31) and obtain

$$
\begin{gathered}
Y=\left[\begin{array}{ll}
9.3310 & 1.7599 \\
1.7599 & 1.8743
\end{array}\right], \\
L=\left[\begin{array}{ll}
2.9293 & 0.6304
\end{array}\right], \quad \gamma=2.0008,
\end{gathered}
$$

then, by Theorem 10 the control law for linear static output feedback can be chosen as

$$
K=0.0505 .
$$

We conclude that the stochastic system (29) is robustly stabilizable by means of static output feedback (30) if we choose $K=0.0505$.

\section{Conclusion}

The problems of robust stability by means of output feedback law for a class of stochastic systems are investigated in this paper. It is shown that the problems can be transformed to the convex optimization problems in the form of linear matrix inequalities. The unified approach is presented in this paper, and how the linear matrix inequalities methods can be used to stabilize the stochastic system is given. The sufficient conditions for the existence of output feedback laws are obtained. Further, we give the numerical example by using the computer simulation to verify the correctness of the theoretical analysis results.

\section{References}

[1] X. R. Mao, "Robustness of stability of stochastic differential delay equations with Markovian switching," Chaos Solitons and Fractals, vol. 45, no. 4, pp. 416-425, 2012.

[2] C. Yuan and X. Mao, "Asymptotic stability in distribution of stochastic differential equations with Markovian switching," Stochastic Processes and their Applications, vol. 103, no. 2, pp. 277-291, 2003.

[3] W.-H. Chen and B.-S. Chen, "Robust stabilization design for nonlinear stochastic system with Poisson noise via fuzzy interpolation method," Fuzzy Sets and Systems, vol. 217, pp. 4161, 2013.

[4] L. Guo and L. Yin, "Robust PDF control with guaranteed stability for non-linear stochastic systems under modelling errors," IET Control Theory \& Applications, vol. 3, no. 5, pp. 575$582,2009$.

[5] M. H. Jiang, Y. Shen, and X. X. Liao, "Robust stability of uncertain neutral linear stochastic differential delay system," Applied Mathematics and Mechanics, vol. 28, no. 6, pp. 829-836, 2007.

[6] H. Yang, H. S. Shu, and X. Kan, "Almost sure stability and stabilization for hybrid stochastic system with interval timevarying delay," Journal of the Chinese Institute of Engineers, vol. 35, pp. 633-640, 2012.

[7] T. Iwasaki and R. E. Skelton, "A unified approach to fixed-order controller design via linear matrix inequalities," Mathematical Problems in Engineering, vol. 1, no. 1, pp. 59-75, 1995.

[8] M. E. Sezer and D. D. Šiljak, "Robust stability of discrete systems," International Journal of Control, vol. 48, no. 5, pp. 2055-2063, 1988.

[9] D. D. Siljak, D. M. Stipanovic, and A. I. Zecevic, "Robust decentralized turbine/governor control using linear matrix inequalities," IEEE Transactions on Power Systems, vol. 17, no. 3, pp. 715-722, 2002.

[10] B. Sari, O. Bachelier, and D. Mehdi, "Robust state feedback admissibilisation of discrete linear polytopic descriptor systems: a strict linear matrix inequality approach," IET Control Theory \& Applications, vol. 6, no. 8, pp. 1097-1108, 2012.

[11] D. D. Šiljak and D. M. Stipanović, "Robust stabilization of nonlinear systems: the LMI approach," Mathematical Problems in Engineering, vol. 6, no. 5, pp. 461-493, 2000.

[12] D. M. Stipanović and D. D. Šiljak, "Robust stability and stabilization of discrete-time non-linear systems: the LMI approach," International Journal of Control, vol. 74, no. 9, pp. 873-879, 2001.

[13] D. W. C. Ho and G. Lu, "Robust stabilization for a class of discrete-time non-linear systems via output feedback: the unified LMI approach," International Journal of Control, vol. 76, no. 2, pp. 105-115, 2003. 
[14] S. Boyd, L. E. Ghaoui, and V. Balakrishnan, Linear Matrix Inequalities in System and Control Theory, Society for Industrial and Applied Mathematics, Philadelphia, Pa, USA, 2007.

[15] F. Jiang, J. Shen, and X. Li, “The LMI method for stationary oscillation of interval neural networks with three neuron activations under impulsive effects," Nonlinear Analysis: Real World Applications, vol. 14, no. 3, pp. 1404-1416, 2013.

[16] X. R. Mao, Stochastic Differential Equations and Applications, Horwood Publishing Limited, Chichester, UK, 2007. 


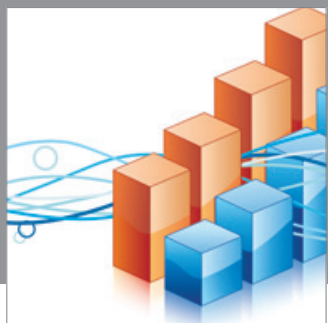

Advances in

Operations Research

mansans

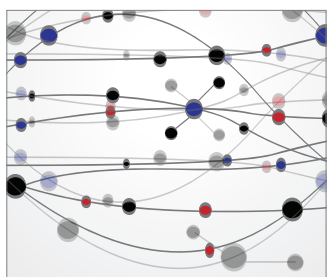

The Scientific World Journal
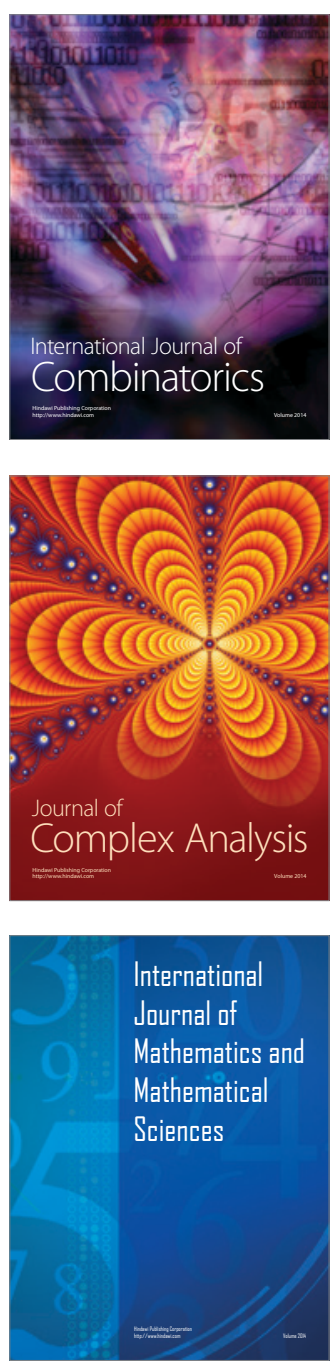
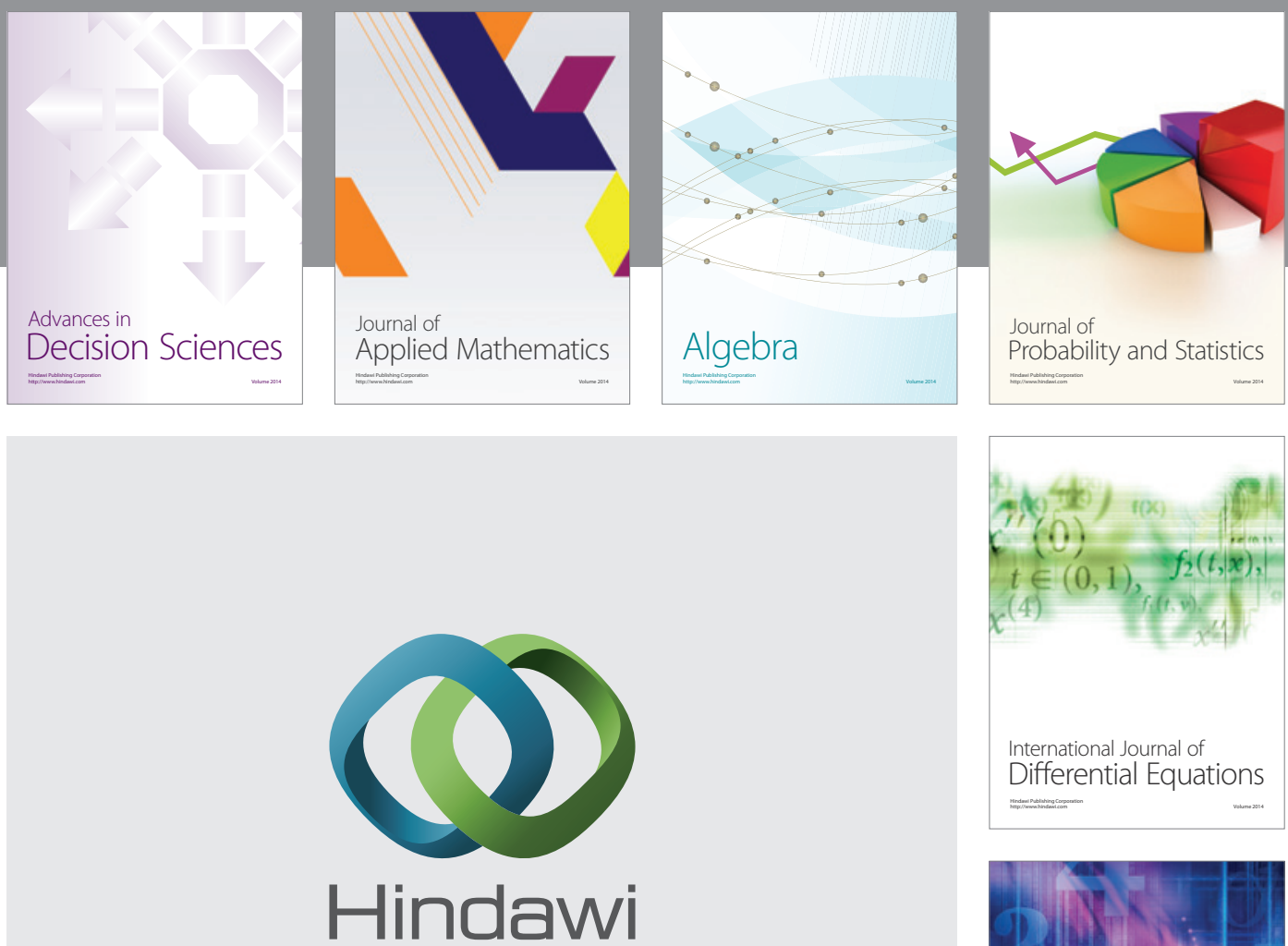

Submit your manuscripts at http://www.hindawi.com
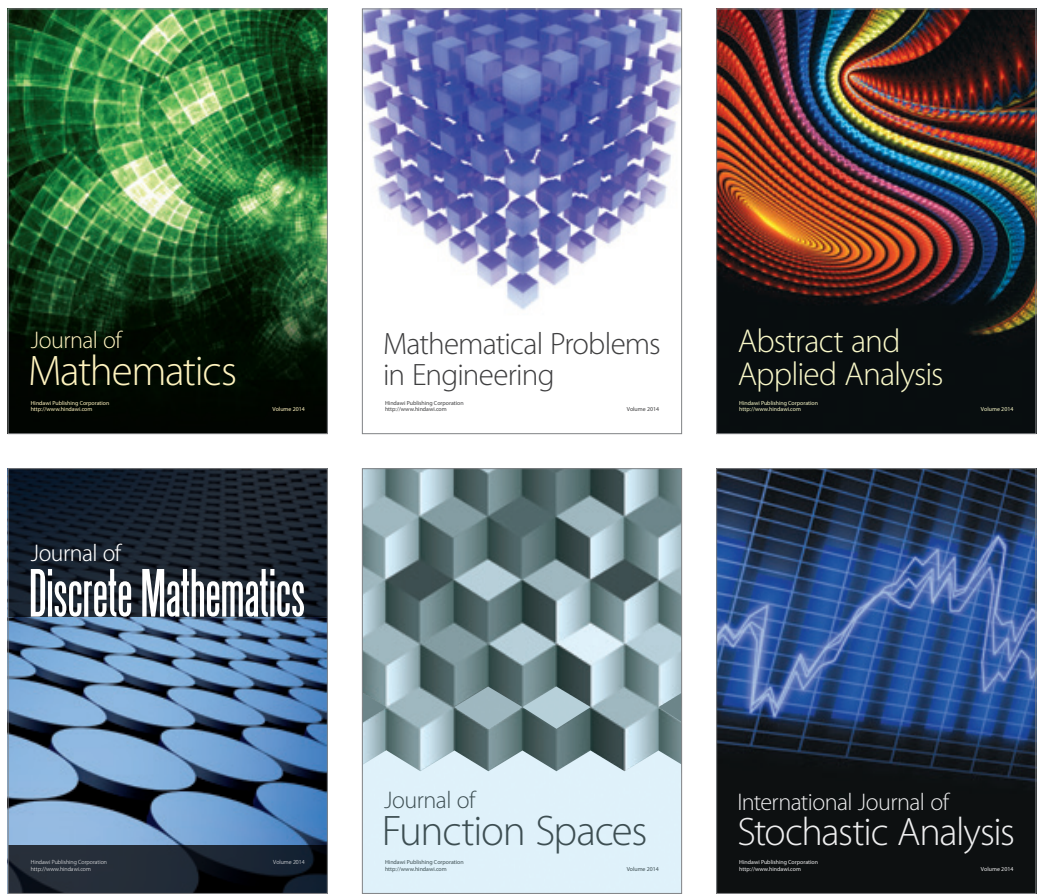

Journal of

Function Spaces

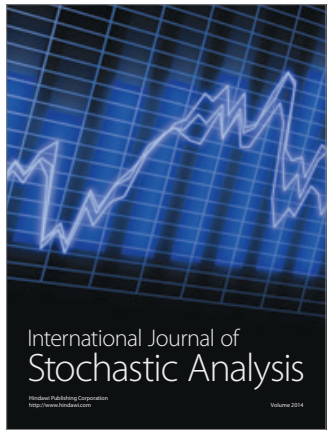

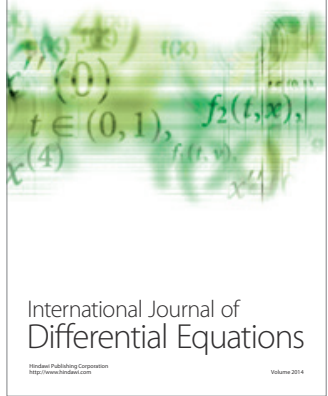
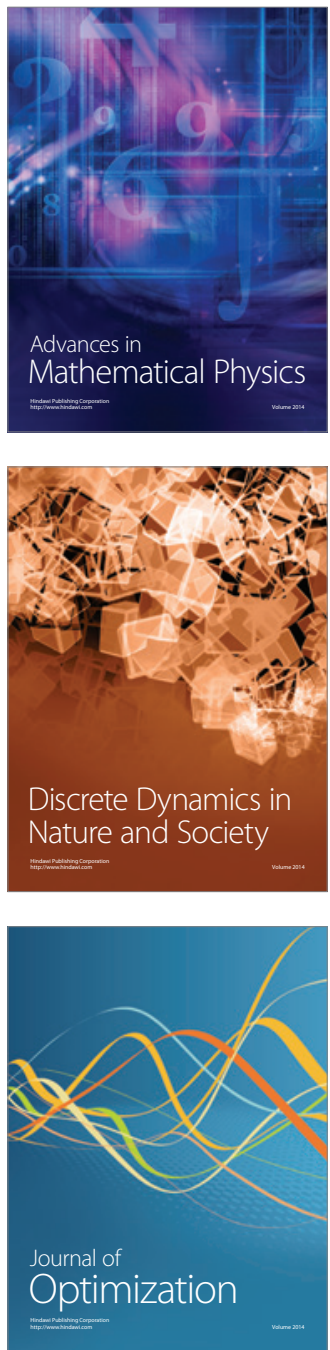